\title{
LA CONSTRUCCIÓN DE VIVIENDA Y EL EMPLEO EN MÉXICO
}

\author{
VÍcTOR L. URQUIDI \\ Adalberto García Rocha * \\ El Colegio de México
}

\section{INTRODUCCIÓN}

Este ARTículo constituye un resumen introductorio a cuatro estudios sobre el empleo en el sector de la construcción de vivienda en México, los cuales se realizaron en el Centro de Estudios Económicos y Demográficos de El Colegio de México, con el patrocinio de la OCDE y la cooperación financiera del Banco Interamericano de Desarrollo, el Banco Internacional de Reconstrucción y Fomento y la Fundación Ford. Los estudios referidos son: Efectos directos $e$ indirectos sobre el empleo en ocho tipos representativos de la vivienda en México, por Christian Araud, con la colaboración de Santiago Rincón Gallardo; Creación de empleo por variación de niveles de tecnología y de producción en la vivienda en México, por Gerard $\mathrm{K}$. Boon; Empleo y alternativas financieras en la vivienda mexicana y La sustitución de trabajo por materiales o capital en la construcción en México, ambos por W. Paul Strassman. Estos cuatro estudios aparecerán en un volumen en preparación.

Simultáneamente, la OCDE ha publicado otro estudio sobre la industria de la construcción ** que se extiende con mayor profundidad sobre las políticas de vivienda en México, los planes de inversión pública y privada, las organizaciones sindicales y patronales en el sector de la construcción, y algunos aspectos de las políticas salariales. Este documento fue elaborado también dentro de la línea de los estudios mencionados.

Los países latinoamericanos se enfrentan al problema agudo de proporcionar empleo a su población activa. En México, el gobierno ha mostrado una preocupación creciente por esta situación, que ha venido haciéndose cada vez más grave. En el cuadro 1 se da información abreviada que permite apreciar la magnitud del problema del desempleo en su dimensión más obvia, esto es, el desempleo abierto. La población activa de México en 1970, según el censo de ese año, fue de casi 13 millones de personas. De ellas, el $4.48 \%$ declaró haber trabajado tres meses o menos en 1969, y el $8.07 \%$ de 4 a 6 meses. Esta última proporción puede con-

* Los autores agradecen al Dr. Montague Yudelman, vicepresidente del Centro de Desarrollo de la OCDE, y a los miembros del grupo consultivo especial (Steering Committee) sus observaciones y sugerencias, que se han tomado en cuenta para elaborar este estudio introductorio.

** Dimitrios A. Germidis, The Construction Industry in Mexico. Technical Papers, Development Centre, OCDE, París, 1972. Llevado a cabo con la coopera ción del Centro de Estudios Económicos y Demográficos de El Colegio de México. 
siderarse como un desempleo abierto equivalente de la mitad, es decir, de $4.03 \%$. En consecuencia, se puede suponer una tasa global de desempleo equivalente de $8.51 \%$, que representa una cifra de 1.1 millones de desempleados tanto urbanos como rurales. En el cuadro 1 se observa además que las proporciones son más graves en el caso de la construcción. En este sector, de acuerdo con el criterio anterior, el desempleo equivalente en 1969 puede estimarse en una proporción de $11.95 \%$, o sea alrededor de 70000 personas.

\section{Cuadro 1}

México: Población económicamente activa y PeRíodos de ocupación, 1969

\begin{tabular}{|c|c|c|c|c|}
\hline $\begin{array}{c}\text { Meses } \\
\text { trabajados }\end{array}$ & $\begin{array}{l}\text { Agricultura, } \\
\text { ganaderia, } \\
\text { ailvicultura, } \\
\text { caza y pesca }\end{array}$ & Construcción & $\begin{array}{c}\text { Otras } \\
\text { actividades }\end{array}$ & $\begin{array}{c}\text { Total } \\
\text { nacional }\end{array}$ \\
\hline De 1 a 3 & $\begin{array}{r}233243 \\
(4.57)\end{array}$ & $\begin{array}{l}35616 \\
(6.24)\end{array}$ & $\begin{array}{r}312 \quad 146 \\
(4.29)\end{array}$ & $\begin{array}{r}581005 \\
(4.48)\end{array}$ \\
\hline De $4: 6$ & $\begin{array}{l}516806 \\
(10.13)\end{array}$ & $\begin{array}{c}65 \quad 182 \\
(11.41)\end{array}$ & $\begin{array}{r}463 \quad 195 \\
(6.36)\end{array}$ & $\begin{array}{r}1045183 \\
(8.07)\end{array}$ \\
\hline De $7: 9$ & $\begin{array}{r}424963 \\
(8.33)\end{array}$ & $\begin{array}{r}65266 \\
(11.43)\end{array}$ & $\begin{array}{r}347757 \\
(4.78)\end{array}$ & $\begin{array}{r}837986 \\
(6.47)\end{array}$ \\
\hline De 10 a 12 & $\begin{array}{rr}3928 & 507 \\
(76.97)\end{array}$ & $\begin{array}{l}404942 \\
(70.92)\end{array}$ & $\begin{array}{r}6157434 \\
(84.57)\end{array}$ & $\begin{array}{r}10 \quad 490883 \\
(80.98)\end{array}$ \\
\hline Total & 5103519 & 571006 & 7280532 & 12. 955057 \\
\hline
\end{tabular}

Fuente: Dirección General de Estadística, IX Censo General de Población, 1970, México, 1972.

Nota: Las cifras entre paréntesis son porcientos respecto del total de las columnas.

a Comprende industria de transformación, comercio, petróleo, servicios, gobierno, etc.

Aunque las cifras anteriores no pueden tomarse como estimación definitiva del volumen del desempleo en México, representan órdenes de magnitud alarmantes. Principalmente en el caso de la construcción, cuya fuerza de trabajo se concentra en el medio urbano, la existencia de tal número de desocupados y su proporción tan elevada explica el porqué del interés por estudiar el problema. Debe mencionarse también que el censo de población de 1970, aun teniendo en cuenta las deficiencias de enumeración, señala que en 1969 el $72 \%$ de la población activa que declaró tener ingresos, manifestó ganar menos de $\$ 1000$ mensuales. Aun cuando exista fuerte sesgo de subdeclaración en esta variable, es posible que la distribución del ingreso se vea reflejada con fidelidad razonable en el porciento anterior; por lo tanto, una estimación de desempleo en función de los niveles del ingreso daría cifras semejantes o tal vez mayores a las anteriores.

En los estudios mencionados se calcula el contenido directo e indirecto de empleo en la construcción de vivienda. ${ }^{1}$ Además de la importancia de los proyectos de construcción de vivienda para satisfacer o atenuar el déficit habitacional, que es creciente, debe tenerse en cuenta la posibilidad de crear empleo por ese medio.

1 Ver los estudios citados de Gerhard K. Boon y Christian Araud. 
Los estudios contienen estimaciones cuantitativas sobre los efectos de un programa determinado de construcción de habitación. Manejan una tipología de vivienda que permite combinar distintas alternativas y derivar sus efectos en la creación de empleo y otros aspectos no menos importantes tales como consumo, inversión, importaciones, etc. Uno de los autores explora también varias opciones tecnológicas que añaden una dimensión que permite la elaboración de estimaciones alternativas. Es importante aclarar que no se pretende calcular el monto óptimo de los recursos nacionales que deben dedicarse al renglón de vivienda en el contexto de un esquema de prioridades de inversión. Tampoco se pretende hacer evaluaciones de la creación óptima de empleo. El objetivo de estos estudios es derivar las "constantes" del proceso en cuanto a la generación de empleo y otros efectos, y queda al lector extender las estimaciones al área de su interés.

La conclusión de esta investigación coincidió con el anuncio del Gobierno Federal, a principios de 1971 , de un nuevo programa para la construcción de vivienda de bajo costo para obreros, ${ }^{2}$ y las cuestiones tratadas aquí coinciden igualmente con los temas que preocupan al gobierno mexicano acerca de las implicaciones económicas de tales políticas. Por ello se espera que estos ejercicios constituyan una ayuda a este respecto y que la utilidad de los trabajos trascienda del interés meramente académico.

Conviene mencionar también que la utilidad de estos trabajos no se limita al caso estudiado. El manejo de la información y los principios metodológicos y teóricos, en la forma en que lo han hecho los autores, es lo bastante flexible para que puedan aplicarse a otras circunstancias, bajo supuestos diferentes, e inclusive con énfasis en aspectos distintos a los presentados aquí. Esto aparece bien ilustrado en los documentos y aumenta su utilidad de aplicación, con tal que se disponga de cierta información básica.

Las investigaciones existentes en México sobre el sector de la construcción de vivienda han sido fragmentarias y de poco alcance; en este sentido, estos trabajos constituyen un intento por hacer un estudio comprensivo del tema. Por otra parte, los estudios de caso sobre empleo son por lo general poco frecuentes en México, salvo algunos enfoques macroeconómicos con énfasis distinto.

Antes de presentar los estudios, se ha considerado conveniente hacer una descripción de aspectos sobresalientes de la construcción de vivienda en el país, con objeto de ubicar al lector dentro de una perspectiva más general, y también para añadir algunas consideraciones sobre el problema de la vivienda y las políticas correspondientes. Tales consideraciones han quedado fuera del alcance de los estudios en sí, y je espera que beneficien al lector y simplifiquen la lectura de los documentos.

Esta descripción previa ha sido dividida en tres partes, en las cuales se examina el déficit de vivienda, las características técnicas del sector de la construcción y por último las implicaciones que los estudios contienen en relación con las políticas de vivienda. Se intenta con esto presentar un panorama global que permita dar respaldo técnico a las conclusiones que se adelantan aquí en relación con la política económica en este campo.

2 Véase más adelante, p. 19. 


\section{El DÉficit DE VIVIENDA EN MÉxico}

Como tantos otros países latinoamericanos, México experimenta un fuerte déficit de vivienda, tanto en el medio urbano como en el rural. Sobre este aspecto, en lugar de efectuar un cálculo más sobre la magnitud del problema habitacional en México, se presenta aquí un resumen de estimaciones hechas por algunos autores y dependencias nacionales, junto con una crítica de las cifras de modo de concluir con una idea sobre el orden de magnitud del número de viviendas faltantes en el país. La razón para proceder así es que la información estadística existente, además de fragmentaria y discontinua, presenta demasiada heterogeneidad en cuanto a las definiciones, $y$ en algunas ocasiones incompatibilidades difíciles de desentrañar.

La única fuente sistemática de información que proporciona datos sobre el acervo de vivienda y las condiciones de ésta son los censos de población. Aparte de éstos, existen publicaciones de varias investigaciones muestrales y estimaciones de algunos autores sobre las distintas componentes del acervo y del déficit. ${ }^{3}$

De ordinario, este déficit se estudia en atención a tres aspectos componentes: a) el hacinamiento que resulta de la acumulación de déficit anteriores y del crecimiento del número de unidades familiares alojadas en viviendas de tamaño fijo; $b$ ) las viviendas necesarias para satisfacer el aumento de la población, y $c$ ) el número de viviendas que es preciso reconstruir parcial o totalmente de acuerdo con normas mínimas sobre su estado de habitabilidad. De las tres categorías anteriores, la menos susceptible de apreciaciones subjetivas parece ser la referente al crecimiento demográfico. En esta categoría, la regularidad estadística de los patrones de tamaño de la familia proporciona un criterio razonable para cuantificar la demanda de vivienda necesaria. De las otras dos componentes, en la de deterioro es indispensable establecer normas para demarcar niveles de habitabilidad; sin embargo, es posible dar una idea del orden de magnitud de cada categoría, de acuerdo con estimaciones de algunos autores y de publicaciones de dependencias oficiales.

Para tener una idea de la composición de la vivienda, véase el cuadro 2, basado en los censos de población de 1950, 1960 y 1970. Puede verse que, de 1960 a 1970 , el número total de viviendas —urbanas y rurales - aumentó en 1.9 millones, que representa un ritmo de creación de alojamientos de 190000 unidades anuales. En este mismo período los censos muestran también un aumento relativo de la cantidad de viviendas propias. En cuanto al tamaño de la vivienda, puede verse que las de 1 a 2 cuartos abarcan la proporción mayor del total (80\% en 1960 y $69 \%$ en 1970); sin embargo, esta última disminución puede ser indicio de que no se ha satisfecho la demanda creciente de habitaciones de 1 a 2 cuartos.

3 Existen varias investigaciones muestrales que han recogido información sobre la vivienda y sus condiciones, y en ellas se apoyan los autores de algunas de las estimaciones que presentamos aquí. Ver, por ejemplo. Instituto Nacional de la Vivienda, Investigación nacional de la vivienda mexicana, 1961-1962, México, 1963, e Instituto Mexicano del Seguro Social, Investigación de vivienda en once ciudades del país, México, 1965. 


\section{Cuadro 2}

MÉxico: Algunas CARACTERísticas DE LAS viviendas EXISTENTES, 1950-1970 (Millones de viviendas)

\begin{tabular}{|c|c|c|c|c|c|c|c|c|}
\hline \multirow[t]{2}{*}{ Años } & \multicolumn{3}{|c|}{ Total de viviendas de } & \multirow{2}{*}{ Urbanas } & \multirow{2}{*}{ Rurales } & \multirow{2}{*}{ Propias } & \multirow{2}{*}{$\begin{array}{c}\text { No } \\
\text { Propias }\end{array}$} & \multirow{2}{*}{ Total } \\
\hline & 1 cuarto & 2 cuartos & más de dos & & & & & \\
\hline 1950 & - & - & - & - & - & $\left(\begin{array}{l}3.4 \\
(64)\end{array}\right.$ & $\begin{array}{l}1.9 \\
(36)\end{array}$ & 5.3 \\
\hline 1960 & $\begin{array}{r}3.5 \\
(55)\end{array}$ & $\begin{array}{l}1.6 \\
(25)\end{array}$ & $(20)$ & $\left(\begin{array}{l}3.1 \\
(48)\end{array}\right.$ & $(52)^{3.3}$ & $\begin{array}{r}3.5 \\
(55)\end{array}$ & $\begin{array}{c}2.9 \\
(45)\end{array}$ & 6.4 \\
\hline 1970 & $\left(\begin{array}{l}3.3 \\
40)^{3}\end{array}\right.$ & $\begin{array}{c}2.4 \\
(29)\end{array}$ & $\begin{array}{r}2.6 \\
(31)\end{array}$ & $\begin{array}{r}4.9 \\
(59)\end{array}$ & $\left(\begin{array}{l}3.4 \\
41)^{2}\end{array}\right.$ & $\begin{array}{l}5.5 \\
(66)\end{array}$ & $\begin{array}{r}2.8 \\
(34)\end{array}$ & 8.3 \\
\hline
\end{tabular}

Fuentes: Dirección General de Estadística, Censos de Población y Vivienda, 1950, 1960 y 1970.

Nota: Las cifras entre paréntesis son porcientos respecto del total. Entre los censos de 1950, 1960 y 1970 hubo cambios en la definición de viviendas propias y no propias, y por tal razón las cifras por este concepto no son estrictamente comparables.

La vivienda tiene otras características cualitativas que convendría señalar, entre otras su estado de habitabilidad. En los cuadros 3 y 4 aparecen algunos indicadores que en algunos casos no corresponden al estado de la vivienda en sí. Sin embargo, sin mucho riesgo de caer en exageraciones, las cifras indican que la vivienda mexicana se encuentra en condiciones deplorables, en atención a que la mayor parte de la población ocupa viviendas que no cumplen con requisitos higiénicos mínimos y vive en condiciones de grave hacinamiento. El 36\% carece de agua entubada y drenaje, el $37 \%$ tiene techos de materiales endebles (palma y teja o similares) y el $41 \%$ tiene piso de tierra. Estas dos últimas categorías pueden corresponder en su mayor parte a viviendas rurales. No se dispone de información para establecer aquí hasta qué punto es así.

\section{Cuadro 3}

MÉxico: Vivienda y ocupantes, SEgún CARACTERÍstica de TECHOS Y PISOS, 1970

(Millones)

\begin{tabular}{|c|c|c|c|c|c|c|c|c|}
\hline & \multicolumn{6}{|c|}{ Material predominante en techos } & \multicolumn{2}{|c|}{$\begin{array}{l}\text { Material predo- } \\
\text { minante en pi } \text { soos }\end{array}$} \\
\hline & Total & $\begin{array}{l}\text { Concreto o } \\
\text { similares }\end{array}$ & $\begin{array}{l}\text { Palma o } \\
\text { similares }\end{array}$ & $\begin{array}{c}\text { Teja o } \\
\text { similares }\end{array}$ & Hadera & Otros & Tierra & Otros \\
\hline$\underset{\not / p}{\text { Vivienda }}$ & $\begin{array}{c}8.3 \\
(100)\end{array}$ & $\begin{array}{l}2.8 \\
(34)\end{array}$ & $\begin{array}{l}1.3 \\
(15)\end{array}$ & $\begin{array}{l}1.8 \\
(22)\end{array}$ & $(11)$ & $\begin{array}{l}1.5 \\
(19)\end{array}$ & $\begin{array}{l}3.4 \\
(41)\end{array}$ & $\begin{array}{l}4.9 \\
(59)\end{array}$ \\
\hline Ocupantes & $\begin{array}{l}48.2 \\
(100)\end{array}$ & $\begin{array}{r}16.4 \\
(34)\end{array}$ & $\begin{array}{l}7.2 \\
(15)\end{array}$ & $\begin{array}{r}10.5 \\
(22)\end{array}$ & $\begin{array}{l}5.2 \\
(11)\end{array}$ & $\begin{array}{l}8.9 \\
(18)\end{array}$ & $\begin{array}{l}19.8 \\
(41)\end{array}$ & $\begin{array}{r}28.5 \\
(59)\end{array}$ \\
\hline $\begin{array}{l}\text { Ocupantes por } \\
\text { viviendas }\end{array}$ & 5.8 & 5.9 & 5.5 & 5.8 & 5.8 & 5.9 & 5.8 & 5.8 \\
\hline
\end{tabular}

Fuente: Dirección de Estadística, IX Censo General de Población, 1970, México, 1972. 
Cuadro 4

MÉxico: Vivienda SEgún CARACTERísticas dE AGUA Y DRENAJE, 1970 (Porcientos)

\begin{tabular}{lccc}
\hline & Con drenaje & Sin drenaje & Total \\
\hline Disponen de acua entuliada & 38.4 & 22.5 & 61.0 \\
Dentro de la vivienda & 31.9 & 6.8 & 38.7 \\
Fuera de la vivienda jero den & & & 10.6 \\
$\quad$ tro del edificio & 5.2 & 5.4 & 11.6 \\
De llave pública o hidrante & 1.3 & 10.3 & 38.9 \\
No disponen de agua entutada & 3.0 & 35.9 & \\
\hline
\end{tabular}

Fuente: Dirección de Estadística, IX Censo General de Población, 1970, México, 1972.

Por otra parte, los datos de 1960 indican que el hacinamiento parece tener una composición que crece linealmente, de modo que los números de viviendas con $1,2,3, \ldots$ ocupantes tienen una distribución que refleja que el hacinamiento está fuertemente concentrado. En el cuadro 5 se observa que el $42 \%$ de las viviendas tiene 6 o más ocupantes, es decir, un número mayor al tamaño medio de la familia en México. Esto denota la existencia de cierto "desperdicio" en cuanto a la distribución de los ocupantes entre las viviendas.

\section{Cuadro 5}

MÉXICO: Distribución DE LA VIVIENDA SEGÚN EL NÚMERO DE OCUPANTES, 1960

\begin{tabular}{ccc}
\hline $\begin{array}{c}\text { Número de ooupantes } \\
\text { por viviendas }\end{array}$ & $\begin{array}{c}\text { Miles de } \\
\text { viviendas }\end{array}$ & $\$$ \\
\hline 1 & 344 & 5 \\
2 & 777 & 12 \\
3 & 871 & 14 \\
4 & 881 & 13 \\
5 & 863 & 12 \\
6 & 760 & 10 \\
7 & 614 & 7 \\
9 & 463 & 8 \\
$10 \mathrm{y}$ más & 311 & 100 \\
Total de viviendas & 525 & \\
\hline
\end{tabular}

Fuente: Dirección General de Estadística, VIII Censo General de Población, 1960, México, 1962.

Veamos ahora las estimaciones del déficit de vivienda, según ha sido calculado en sus tres componentes y en varios puntos del tiempo. Se han mencionado las tres componentes de hacinamiento, deterioro y crecimiento demográfico, y parece conveniente dar una idea breve de la definición de cada una de ellas. Si se divide la población total entre una estimación del tamaño medio de la familia ( 5.4 personas), se obtiene el total de familias en el país. Si cada familia ha de tener su casa, la diferencia 
con el número de viviendas existentes constituye el déficit por hacinamiento.

Deberá reconocerse, sin embargo, que el cálculo de una cifra promedio a nivel nacional oculta el problema que representa el significado de la vivienda para los distintos estratos sociales, lo que afecta las estimaciones que puedan hacerse del déficit.

Otra manera de calcular este tipo de déficit consiste en establecer una norma en cuanto al número de personas por cuarto y compararla con el promedio existente, haciendo las debidas subdivisiones urbana, rural, vivienda por tamaño, etc. Esto quiere decir que, por ejemplo, si la vivienda debe tener en promedio dos ocupantes por cuarto, entonces debe construirse el número de habitaciones necesario para alojar a las familias de $1,2,3$, etc., miembros, según la distribución de tamaños de las familias. Debe aclararse que este método implica un conocimiento de la distribución de la población por tamaños de familia, de modo de poder traducir el número de cuartos necesarios a número de viviendas. En la columna 3 del cuadro 6 aparecen algunas estimaciones de varios autores, que se han calculado por uno $\mathrm{u}$ otro de los procedimientos anteriores. Puede verse que la variación de las dos estimaciones va de 700000 a 2100000 unidades.

Veamos ahora estimaciones del déficit a cubrir por concepto del crecimiento de la población. Aquí, el cálculo se hace simplemente dividiendo el crecimiento demográfico esperado por el tamaño medio de la familia y se obtiene así el número de viviendas necesarias. ${ }^{4}$ Véase para ello la columna 4 del mismo cuadro 6 . En este caso, las estimaciones giran alrededor de 300000 viviendas anuales en la actualidad.

En el caso de las viviendas deterioradas, o viviendas deleznables, las estimaciones siguen un criterio más arbitrario. Por ejemplo, el Banco Nacional de Obras y Servicios Públicos considera que es necesario reponer alrededor del $30 \%$ de las viviendas hechas de adobe, el $10 \%$ de las de tabique, el $50 \%$ de las de madera, el $100 \%$ de las de embarro y el $5 \%$ de las hechas con otros materiales. ${ }^{5}$ Otros criterios menos elaborados establecen simplemente una proporción de viviendas en los medios rural y urbano como montos de viviendas por reconstruir. ${ }^{6}$ Estos porcientos pueden referirse a las viviendas con techos ruinosos, muros ruinosos, etc. Las cifras que aparecen en la columna 5 del cuadro mencionado son estimaciones de esta componente del déficit, y varían entre 1000000 y 1900000 .

El déficit total, con las divergencias de tamaño que se han observado en las distintas componentes, viene a resultar con una latitud muy amplia, como ilustra la última columna del cuadro 6. Puede estimarse que en la actualidad varía entre 2300000 y 4000000 , y alrededor de dos tercios es urbano. Según una de las estimaciones, en 1980 tal déficit alcanzaría la cifra de 6900000 , o sea el $75 \%$ superior a lo estimado para 1969.

Con estas estimaciones del déficit resulta prácticamente imposible lle-

4 En este caso sería preciso conocer también la distribución de las familias por tamaño para obtener una estimación más refinada.

5 Estimaciones citadas en Secretaría de la Presidencia, Programa nacional de vivienda, México, 1970 (mimeografiado).

- Este es el caso de las estimaciones de J. Puente Leyva, "El problema habitacional", en El Perfil de México en 1980, Vol. 2, México, Siglo XXI, 1972. 


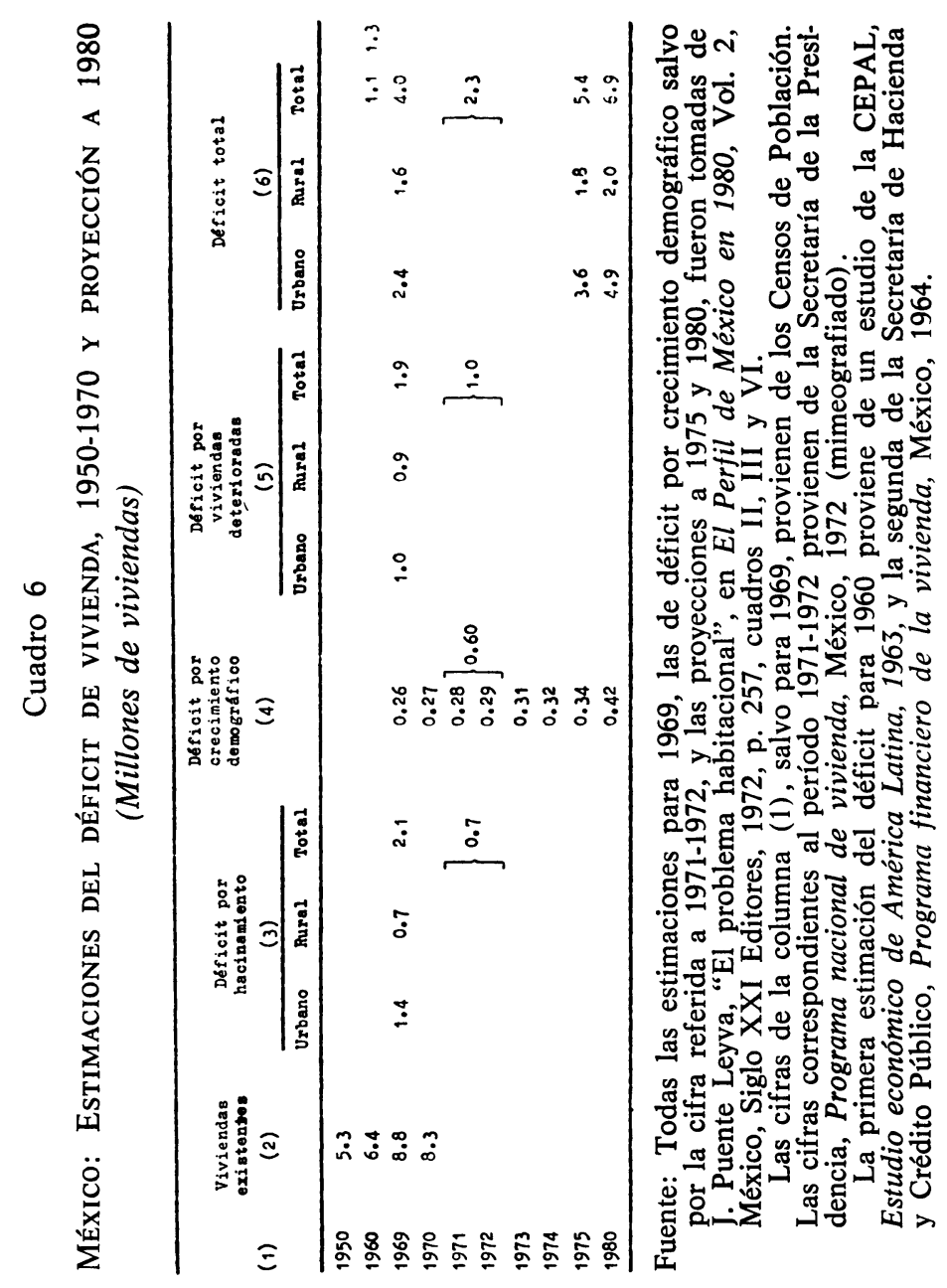


gar a una conclusión útil para implementar una política habitacional masiva. De cualquier manera, la comparación de la estimación más modesta del faltante con el número de viviendas que se construyen anualmente, inclusive en períodos en los que se ha dado impulso a la vivienda, lleva a la conclusión inevitable de que en el país se acumula un faltante de vivienda a una velocidad muy elevada. En el período 1965-1969, la banca privada otorgó créditos para la construcción de alrededor de 58000 viviendas de interés social al año, de un total de viviendas construidas de alrededor de 92000 anuales. ${ }^{7}$ De este modo, tales cifras de construcción representan apenas la tercera parte del déficit por crecimiento demográfico solamente. Esta circunstancia se concentra además en el medio urbano dado el auge de la migración campo-ciudad, que ha transformado la estructura urbana del país a un ritmo notable: en 1950 el $28.0 \%$ de la población vivía en localidades mayores de 15000 habitantes, contra $44.9 \%$ en $1970 .^{8}$

Las cifras permiten llegar a la afirmación de que tan sólo para detener la acumulación del déficit es necesario construir al menos alrededor de 250000 a 300000 viviendas anuales. Es a partir de este volumen de donde la construcción adicional empezaría a atenuar el déficit acumulado, si a esta consideración no se añade la tasa de deterioro anual de las viviendas existentes.

La solución de este problema supone, por fuerza, la necesidad de conocer los rasgos económicos más salientes de la construcción de vivienda en cuanto al proceso productivo en sí. En este aspecto, este sector tiene el rasgo notable de ser clave en la creación de empleo, como se procura mostrar en los párrafos siguientes.

\section{El SECTOR DE LA CONSTRUCCIÓN DE VIVIENDA EN MÉxico}

La intención de esta sección es utilizar los resultados de los trabajos mencionados en la introducción y otras piezas de información para describir en forma global fases fundamentales de la construcción de vivienda, como son la organización de los factores productivos, la tecnología y algunos aspectos del mercado, vistos desde su ángulo financiero. Con esto se espera poner de relieve algunos problemas básicos para la política económica del sector.

Por muchas razones que se enumeran en seguida, el sector de la construcción (de vivienda y no vivienda) tiene importancia económica especial. Por una parte, las componentes de instalaciones industriales e infraestructura económica desempeñan un papel medular en el crecimiento económico de la mayoría de las actividades. A esto se une el hecho de que el sector público es el principal constructor de infraestructura y por tal razón es a través de la construcción como la inversión pública juega su papel económico. La inversión pública abarca también la construcción de un monto considerable de obras de beneficio social. En el sexenio 1964-1970 se gastaron $\$ 32000$ millones en obras de bienestar social, que

7 Secretaría de Hacienda y Crédito Público, FOVI y FOGA, Programa financiero de la vivienda; evaluación y proyección, México, 1970, pp. 64 y 69.

8 Ver Centro de Estudios Económicos y Demográficos, Dinámica de la pobla ción de México, México, El Colegio de México, 1970, cuadro V-10, p. 131. La cifra de 1970 se basa en el censo de este año. 
representaron el $25 \%$ de la inversión federal total; a la vivienda se destinaron $\$ 2000$ millones. La construcción de obras de infraestructura de transportes y comunicaciones representó a su vez una partida semejante de $\$ 20000$ millones. En consecuencia, estos renglones, eminentemente de la construcción, abarcan alrededor de la mitad de la inversión federal. ${ }^{9}$ Las partidas restantes incluyen igualmente una cantidad sustancial de construcción de instalaciones industriales.

En México, el sector de construcción e instalaciones representó en 1965 el $7 \%$ del producto bruto interno. ${ }^{10} \mathrm{El}$ sector de la construcción se divide como sigue: $31 \%$ en vivienda y $69 \%$ en otras construcciones e instalaciones en términos de valor total de la producción. ${ }^{11}$ Como sector individual, es de los más importantes desde el punto de vista del empleo, en especial de trabajadores no calificados. El coeficiente de generación global de empleo de este sector por unidad de gasto en el mismo es de los mayores, ${ }^{12}$ y un gasto en la demanda final de vivienda desencadena un monto de producción en toda la economía de un poco más del doble de aquél. ${ }^{13}$ La construcción es, pues, uno de los generadores de empleo más poderosos en la economía.

En cuanto a la distribución del ingreso, los salarios pagados en el sector de la construcción representaron en promedio el $8 \%$, en 1967, del total nacional de sueldos, salarios y prestaciones. En las cuentas nacionales ${ }^{14}$ se observa que la distribución del ingreso se ha sesgado en favor de las utilidades, las cuales, de 1950 a 1967, crecieron a una tasa media anual de $11.7 \%$, contra $4.6 \%$ de los salarios pagados en el sector, es decir, a menos de la mitad de la primera de estas tasas. Por el lado de la composición de los pagos a los factores, el cambio ha sido notable: en 1950 , los salarios representaban el $80 \%$ del valor agregado y para 1967 la relación se había reducido a $48 \%$ (a precios de 1960). Este fenómeno muestra que en algún lugar del proceso productivo, probablemente en la política de capitalización, han ocurrido ajustes que han contrarrestado las políticas de salarios mínimos y han deteriorado la distribución del ingreso. El renglón de la construcción, precisamente por el papel del gasto y la política públicos y su influencia subsecuente sobre el ritmo de inversión privada, sufre fluctuaciones que se manifiestan en el empleo. El volumen anual de construcción resulta así una variable medular en el empleo y sus fluctuaciones.

La mano de obra de la construcción tiene características especiales. ${ }^{15}$ En el fenómeno de migración campo-ciudad, que se observa ahora en magnitudes impresionantes en algunas ciudades de México, la construcción - principalmente la de vivienda y obras urbanas- desempeña un papel clave al dar al migrante una ocupación transitoria accesible a sus bajos niveles de calificación; se genera así un mecanismo de retención

9 Secretaría de la Presidencia, Inversión pública federal 1965-1970, México. 1970 , pp. 39 ss.

10 Véase Banco de México, Cuentas nacionales y acervos de capital, 1950-1967.

11 Véase G. K. Boon, op. cit., Cap. 4.

12 Véase C. Araud, op. cit., apéndice del Cap. I.

13 Véase Boon, op. cit.

14 Con base en Banco de México, Cuentas nacionales y acervos de capital, 1950-1967, México, 1969. Los porcientos expresados en este párrafo provienen del trabajo de W. Paul Strassman, La sustitución de..., citado al principio.

${ }_{15}$ Para un estudio más completo, véase el trabajo de D. Germidis, antes mencionado. 


\section{Cuadro 7}

NÚMERO TOTAL DE DEPENDIENTES DE OBREROS DE LA CONSTRUCCIÓN EN UNA muestra de México, D. F., Guadala jara, Monterrey, Tampico Y VERACRUZ, 1971

\begin{tabular}{|c|c|c|c|c|}
\hline Ocupación & $\begin{array}{l}\text { De } 1-4 \text { depen } \\
\text { dientes }\end{array}$ & uás de 4 & $\begin{array}{l}0 \text { dependientes } \\
\text { y no respuesta }\end{array}$ & Total \\
\hline Peones & $\begin{array}{l}134 \\
(14)\end{array}$ & $\begin{array}{l}49 \\
(5)\end{array}$ & $\begin{array}{l}140 \\
(15)\end{array}$ & $\begin{array}{l}323 \\
(34)\end{array}$ \\
\hline Maestros & $(5)$ & $\begin{array}{l}66 \\
(7)\end{array}$ & $\left(\begin{array}{c}17 \\
2\end{array}\right)$ & $\begin{array}{l}131 \\
(14)\end{array}$ \\
\hline Oficiales & $\begin{array}{l}124 \\
(13)\end{array}$ & $\begin{array}{c}90 \\
(10)\end{array}$ & $\left(\begin{array}{c}77 \\
8\end{array}\right)$ & $\begin{array}{l}291 \\
\text { (31) }\end{array}$ \\
\hline $\begin{array}{l}\text { Operadores, trabajadores - } \\
\text { de obra metálica y otros }\end{array}$ & $\left(\begin{array}{c}85 \\
9\end{array}\right)$ & $\left(\begin{array}{c}65 \\
7\end{array}\right)$ & $\left(\begin{array}{c}49 \\
5\end{array}\right)$ & $\begin{array}{l}199 \\
(21)\end{array}$ \\
\hline Total & $\begin{array}{l}391 \\
(41)\end{array}$ & $\begin{array}{l}270 \\
(29)\end{array}$ & $\begin{array}{l}283 \\
(30)\end{array}$ & $\left(\begin{array}{l}944 \\
(100)\end{array}\right.$ \\
\hline
\end{tabular}

Fuente: Encuesta especial a obreros de la construcción, que forma parte de un proyecto de investigación de sindicatos, obreros y patrones en el sector de la construcción, llevado a cabo por D. Germidis, para el Centro de Desarrollo de la OCDE con la cooperación del Centro de Estudios Económicos y Demográficos de El Colegio de México.

Nota: Se han eliminado algunas observaciones por la mala calidad de la respuesta a esta pregunta.

al menos temporal en el medio urbano. Por la naturaleza de las obras, el obrero de la construcción está sujeto a una rotación muy elevada que lo coloca en una posición precaria que involucra períodos de desempleo sustanciales entre una ocupación y otra. Este fenómeno ha viciado los mecanismos de contratación y puede ser un factor explicativo del deterioro de la distribución del ingreso mencionado antes. Con el obrero de la construcción el patrón no contrae compromisos a largo plazo, y con mucha frecuencia el trabajador carece de prestaciones sociales. A este respecto, cabe mencionar el hecho de que este trabajador tiene el rasgo socioeconómico adicional de que el tamaño de la familia, en términos del número de dependientes, es considerable. El número de personas beneficiadas con el ingreso de este obrero puede apreciarse en el cuadro 7 , tomado de una muestra de obreros de construcción de vivienda y obras urbanas en cinco ciudades del país en 1971: el 29\% de los trabajadores tiene más de 4 dependientes. La elevada tasa de crecimiento demográfico en el país, a razón de $3.3 \%$ anual entre 1950 y 1970, explica en buena medida el fenómeno de migración-baja calificación-bajos salarios-bajo poder de contratación, que caracteriza al grueso de la fuerza de trabajo de la construcción. La tasa de natalidad de las áreas rurales se estima en 50 al millar. La mortalidad general ha descendido de 17.0 a 9.4 al millar entre 1945-1949 y 1965-1967. ${ }^{16}$

Técnicamente, la construcción es una de las ramas que tienen ante sí una amplia variedad de opciones tecnológicas. Tres fases del proceso de

16 Véase Centro de Estudios Económicos y Demográficos, op. cit., caps. II, III y VIII. 
construcción parecen ser clave en este sentido: excavación, mezclado de cemento y transporte vertical de materiales dentro de la obra. ${ }^{17}$ Esto obliga a considerar cuidadosamente el papel de los precios relativos de los factores capital y trabajo en cuanto a la decisión del constructor sobre la forma de producir. La elasticidad de sustitución del modelo neoclásico, evaluada sobre el supuesto de que la sustitución ocurre a través de los materiales, tiene un valor cercano a la unidad, lo que significa un grado de respuesta sustancial a cambios en dichos precios relativos. ${ }^{18}$ Aquí puede encontrarse un elemento más que explique las fluctuaciones en la distribución de los pagos a los factores. El mecanismo de sustitución opera al decidir el empresario utilizar materiales que requieren menos mano de obra en el sitio de la construcción, como respuesta a un "encarecimiento" relativo del factor trabajo, que puede tomar la forma de mayores salarios o de fricciones laborales de alguna otra índole que obstaculicen el uso de mano de obra. La sensibilidad de la respuesta según el mecanismo anterior se refuerza por el hecho de que la participación de la nómina en el presupuesto de las obras es sustancial (de $37 \%$ en promedio) ${ }^{19}$ y por ende son importantes las decisiones de costo a este respecto. El uso de los materiales está por supuesto determinado en parte por los gustos del comprador respecto del tipo de vivienda que desee adquirir. En resumen, la elección de tecnología y tipos de producto sirven al empresario como mecanismo de escape a los aumentos de los salarios relativos.

Los precios de la construcción muestran fuertes disparidades geográficas, y en esta circunstancia intervienen el costo del trabajo en la construcción misma y el contenido en los materiales utilizados. Queda fuera del alcance de este estudio tratar el punto, pero sería interesante estudiar la hipótesis de que los costos de la construcción guardan una asociación estrecha con las diferencias en los costos de la mano de obra directa y la contenida en los materiales. Se podrían así explicar en parte las disparidades de los costos.

En la adquisición de vivienda, los canales financieros participan de manera determinante. La elevada densidad económica de este producto lo dejan fuera del alcance del consumidor ordinario, que no puede adquirirlo en forma independiente; por tal razón las instituciones bancarias e hipotecarias son factores clave. Los criterios para establecer las contribuciones de precios, plazos, pagos iniciales, tasas de interés, etc., son elementos a considerar en la explicación del funcionamiento del mercado, y en consecuencia sobre la creación de empleo en la construcción de vivienda. Las posibilidades de incorporar más consumidores potenciales dependen ciertamente de estos elementos $\mathrm{y}$, por ejemplo, la flexibilidad de las "facilidades de pago" en la vivienda podría añadir alrededor de 914000 consumidores de ingresos bajos dentro del estrato de $\$ 701$ a $\$ 950$ mensuales ${ }^{20}$ si se estableciera una política de subsidio que manejara formas de financiamiento, de modo que la participación del gasto en vivienda en la canasta de bienes de este estrato de población permaneciera constante.

17 Véase Boon, op. cit., cap. III.

18 Véase Strassman, La sustitución de trabajo por..., op. cit.

19 Derivado de información recopilada por Araud, op. cit.

20 Véase W. Paul Strassman, Empleo y alternativas financieras en la vivienda mexicana, trabajo antes mencionado. Aunque los cálculos del autor están ejemplificados con una vivienda de $\$ 20000$, el argumento sigue siendo válido. 
Volviendo al tema del empleo, se presentan y examinan ahora algunos aspectos cuantitativos tomados de los estudios arriba mencionados. Dos de estos estudios guardan estrecha relación en virtud de que el centro de sus estimaciones es una tipología de vivienda elaborada de modo de tener una escala representativa del mercado de vivienda. ${ }^{21}$ Los tipos fueron diseñados en función de las prácticas más usuales en la construcción, y mediante el análisis de las estructuras de costos de una muestra de obras. Estos tipos están clasificados en términos de tamaño y calidad, y su representatividad es analizada en uno de los trabajos." Algunos aspectos de esta tipología se muestran en el cuadro 8.

Cuadro 8

MÉXICO: CARACTERÍSTICAS DE LOS TIPOS DE VIVIENDA

\begin{tabular}{|c|c|c|c|c|c|c|}
\hline \multirow{2}{*}{$\begin{array}{c}\text { Tipo } \\
\text { de } \\
\text { vivienda }\end{array}$} & \multirow{2}{*}{$\begin{array}{l}\text { Superficie } \\
\text { construldáa } \\
\text { media }\left(\mathrm{m}^{2}\right)\end{array}$} & \multirow{2}{*}{$\begin{array}{l}\text { Precio de } \\
\text { venta con } \\
\text { terreno }\end{array}$} & \multirow{2}{*}{$\begin{array}{l}\text { Precio de } \\
\text { venta sin } \\
\text { terreno }\end{array}$} & \multirow{2}{*}{$\begin{array}{c}\text { Número de } \\
\text { viviendas } \\
\text { existentes } \\
\text { en cada } \\
\text { tipo } \\
\end{array}$} & \multicolumn{2}{|c|}{$\begin{array}{l}\text { Empleo total anual } \\
\text { en años-tombre }\end{array}$} \\
\hline & & & & & $\begin{array}{l}\text { Por unidad } \\
\text { media de } \\
\text { vivienda }\end{array}$ & $1 \stackrel{\text { Por } \mathrm{m}^{2}}{200}$ \\
\hline Unifamiliar & & & - & 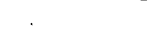 & & \\
\hline Menima & 47.3 & 28.8 & 19.2 & 1144024 & 0.75 & 16.67 \\
\hline De kajo costo & to 71.6 & 64.4 & 47.2 & 804005 & 1.78 & 25.77 \\
\hline Mediano & 163.9 & 207.0 & 153.1 & 225883 & 5.48 & 35.46 \\
\hline De lujo & 384.6 & 702.6 & 484.6 & 29397 & 15.79 & 48.25 \\
\hline \multicolumn{7}{|l|}{ Multifaniliar } \\
\hline De kajo costo & to 67.3 & 47.9 & 43.8 & 252334 & 1.70 & 25.52 \\
\hline $\begin{array}{l}\text { Hediano, } 5 \text { pi } \\
\text { sos o menos }\end{array}$ & 89.7 & 97.2 & 88.0 & 75000 & $3 \cdot 10$ & 35.31 \\
\hline $\begin{array}{l}\text { Medrano,major } \\
\text { de } 5 \text { pisos }\end{array}$ & 89.7 & 119.7 & 109.7 & & 3.70 & 41.94 \\
\hline \multirow[t]{2}{*}{ De Iujo } & 112.1 & 230.7 & 207.9 & $32 \quad 443$ & 6.24 & 57.08 \\
\hline & & & & 2563786 & & \\
\hline
\end{tabular}

Fuentes: C. Araud, Efectos directos $e$ indirectos sobre el empleo en ocho tipos de vivienda en México, op. cit. y G. K. Boon, Creación de empleo por variación de tecnología y de producción en la vivienda en México, op. cit.

a Miles de pesos.

En este cuadro, la última columna representa el contenido de empleo por $1000 \mathrm{~m}^{2}$ de construcción de los tipos correspondientes en 1965. En estas cifras se advierte una progresión clara en el volumen de empleo total, directo más indirecto, a medida que se pasa de las unidades mínimas y de bajo costo a las medianas y de lujo. El empleo directo es aquel que corresponde al pago de salarios en la construcción en sí incluida la infraestructura urbana, o sea el empleo en la obra y en las actividades accesorias. Por su parte, el empleo indirecto es el contenido en la producción de los materiales de construcción y en el consumo de capital. La metodología utilizada es la del modelo de insumo-producto, mediante el

21 Véanse los detalles en el trabajo de Araud, op. cit., Cap. I.

22 Véase Boon, op. cit., Cap. 4. 
cual se ha calculado un conjunto de coeficientes que miden el monto de empleo indirecto por unidad de empleo directo en cada sector de actividad. El empleo directo se calculó mediante un estudio de costos que abarcó además el cálculo de coeficientes técnicos específicos en un conjunto de 16 subsectores económicos que suministran los insumos de la construcción. En este conjunto están contenidos los costos de la vivienda en sí (costo directo), la infraestructura técnica y la promoción. Los coeficientes de empleo indirecto estiman la propagación del efecto a toda la economía. ${ }^{23}$

Para cada uno de los tipos de vivienda, la composición relativa del empleo en directo e indirecto tiene la forma que aparece en las cifras entre paréntesis en la parte extrema izquierda del cuadro 9, donde se advierte que el empleo directo disminuye a medida que aumenta el costo de la vivienda por metro cuadrado construido. Como se verá en seguida, esto quiere decir que las viviendas más baratas son las que tienen efecto global más fuerte sobre la distribución del ingreso si se toma en cuenta que los salarios del obrero ocupado en el sitio de la construcción son los más bajos de todos, y que estas obras baratas son las que hacen menos 'so de equipo. Si el empleo se genera en el sector de vivienda en sí, en lugar de generarse indirectamente en los otros sectores, la derrama de salarios ocurrirá en categorías ocupacionales más bajas. Aunque este mecanismo merece ser investigado más a fondo, puede adelantarse que el empresario, al utilizar materiales caros, eleva el costo de la vivienda y el empleo se genera en estratos de calificación mayores donde la mano de obra es más cara y en sectores donde probablemente es más alta la intensidad del uso del capital. En buena medida, desde luego, la composición de la construcción de vivienda por tipo está dictada por el consumidor, y el uso de ciertos materiales obedece más a esta circunstancia. Sin embargo, podemos observar que el desplazamiento del empleo directo hacia el indirecto es de magnitud relativamente pequeña; según indica el cuadro 9, la variación es de por los menos 17 puntos de porciento entre los tipos extremos de unifamiliar mínimo y multifamiliar de lujo. Se advertirá que el empleo aumenta sistemáticamente a medida que el precio de la unidad hace otro tanto, cuando no se aísla el efecto del tamaño de la vivienda.

En otro de los estudios se utiliza el modelo de insumo-producto para estimar la propagación de un aumento de la demanda final de construcción de vivienda al resto de la economía. Esto se hace a través de un conjunto de coeficientes técnicos estimados para cada uno de los tipos de vivienda diseñados en el primer estudio. El procedimiento consiste esencialmente en desglosar el renglón de construcción, estimar los coeficientes técnicos para cada tipo, y resolver el modelo en función de la demanda final. En seguida, un conjunto de relaciones trabajo/producto llevan a una estimación del empleo en cada sector. Las estimaciones cubren cuatro alternativas tecnológicas y una de costo directo. Las distintas tecnologías provienen de consideraciones sobre la forma de realizar los procesos de excavación, mezclado de cemento y transporte vertical de los materiales. La distinción entre empleo directo e indirecto se hace en este caso

23 Véanse los detalles en Araud, op. cit., apéndice al cap. I. 


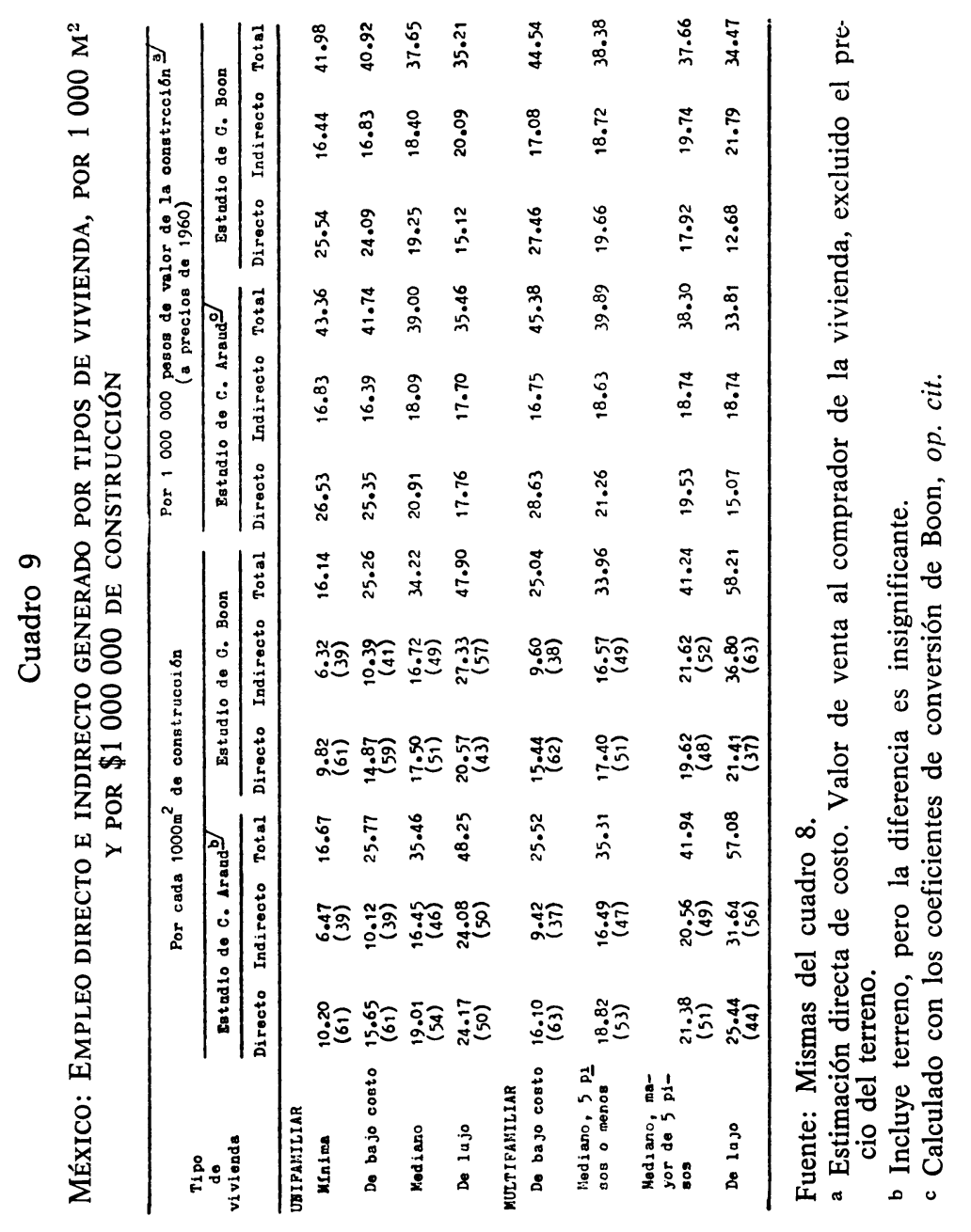


conforme a la definición usual del modelo de insumo-producto en términos de la expansión de potencias del modelo. ${ }^{24}$

En el cuadro 9 aparecen las estimaciones de la creación de empleo en 1965, por tipo de vivienda, incluida la infraestructura urbana, según cada uno de los autores mencionados, y por cada $1000 \mathrm{~m}^{2}$ de área construida y por millón de pesos de gasto en cada tipo, a precios de 1960 . En las estimaciones por unidad de superficie se aprecia el aumento de la creación de empleo al elevarse la calidad de la vivienda, así como que la composición del efecto empleo en directo e indirecto cambia al mejorar dicha calidad. La semejanza de los resultados es notable, y sobre el punto de la división de efectos, las cifras reflejan el hecho de que, al aumentar el costo de vivienda, el empleo se desplaza hacia los materiales. En el caso de la estimación de Araud este desplazamiento es de 11 puntos de porciento en favor del empleo indirecto en viviendas unifamiliares, y de 19 puntos en el caso de las multifamiliares. En el caso de las estimaciones de Boon el desplazamiento es mayor: 18 puntos en las unifamiliares y 25 en las multifamiliares.

En la parte derecha del cuadro 9 se observa que los tipos de vivienda que generan más empleo son los unifamiliares mínimo y de bajo costo, tanto en el caso de Araud (43.36 y 41.74 hombres-año) como en el de Boon (41.98 y 40.92 hombres-año), y el multifamiliar de bajo costo (45.38 en el caso de Araud y 44.54 en el caso de Boon). Esto significa que la vivienda más barata es la que genera más empleo, alrededor de 41 a 45 hombres-año por millón de pesos gastados en su construcción. De este empleo, entre 24 y 28 hombres-año se ocupan en la obra misma y entre 16 y 17 se generan en el resto de la economía. Debe subrayarse que el gasto de un millón de pesos está referido a precios del año de 1960. En otras palabras, el millón de pesos representa en la actualidad una erogación nominal mayor, en función de la elevación de los precios, por lo cual las estimaciones que se hagan con las cifras referidas deberán estar debidamente ajustadas. ${ }^{25}$

De las cifras anteriores queda bien clara la conclusión. Los tipos de vivienda que generan más empleo son los de más bajo precio al consumidor. Estos tipos generan el máximo del empleo tanto en términos del proceso de construcción como en términos del mercado de vivienda, es decir, las posibilidades de ampliar el número de consumidores potenciales son obviamente mayores que en el caso de otros tipos más caros. El tipo multifamiliar de bajo costo, además de generar más empleo en comparación con cualquiera de los siete tipos restantes, es tal vez el de mayores posibilidades en el mercado, en virtud de que pueden lograrse ahorros importantes en relación con el precio del terreno y las superficies necesarias para edificación.

Las diferencias entre las estimaciones de los dos autores son de pequeña magnitud global (alrededor de un hombre-año), aunque son importantes en cuanto a la composición en empleo directo e indirecto. Esto se debe a diferencias en la definición de estos conceptos y en el manejo del modelo de insumo-producto utilizado por los autores. De cualquier manera, ambas estimaciones conducen a la misma conclusión práctica.

En las otras variables, el efecto de los gastos en construcción es im-

24 Véase Boon, op. cit.

25 Ibid. 
portante. En el caso del valor de la producción total, una unidad de demanda final en la vivienda requiere de una producción global de un poco más del doble en toda la economía. El efecto-valor de la producción se reparte aproximadamente en $48 \%$ en el sector de vivienda mismo y el $52 \%$ en el resto de la economía.

En el caso de la inversión, el efecto es aún más notable y ciertamente de la mayor importancia. Una unidad de demanda final en vivienda solicita de la economía necesidades de inversión global de alrededor de 2.3 unidades. ${ }^{26}$ Esto da idea de la presión que la vivienda puede colocar sobre el aparato productivo, y de la necesidad de contar con una oferta flexible en los demás sectores. Este efecto puede reflejar también la necesidad de adoptar alguna modificación en la tecnología de las industrias accesorias a la de construcción de vivienda, de modo de aligerar un poco estas necesidades de inversión y a la vez crear más empleo.

En cuanto al renglón de importaciones, el efecto de una unidad de gasto en vivienda es de alrededor de 0.29 unidades de requerimientos de importación de todo tipo (consumo intermedio e inversión). En otras palabras, 1 millón de pesos de gasto en vivienda genera necesidades del orden de 290000 pesos de importación total. ${ }^{27}$ Los requisitos de importación mayores son los de bienes de capital (68\%), mientras los bienes de consumo ocupan una proporción menor $(32 \%)$. Sobre estas estimaciones debe aclararse que las relaciones inversión/producto utilizadas para el cálculo no tienen en cuenta el grado de utilización de la capacidad productiva. Por esta razón, las cifras pueden representar una sobreestimación, aunque es posible que en algunos sectores exista falta de capacidad y que en el conjunto este sesgo se contrarreste en alguna medida. La estimación de este efecto requiere de un análisis más a fondo.

\section{Aspectos de la política de construcción de vivienda}

Se harán ahora algunos comentarios acerca de las implicaciones de los resultados de los estudios aludidos y de algunas otras evidencias sobre la política de construcción habitacional masiva. Estos comentarios pueden resultar también aplicables al caso de otros países que proyecten programas de vivienda con objeto de crear empleo y atenuar el déficit. No es que los resultados ilustren cuestiones realmente nuevas, sino que se pretende mostrar en estas líneas la utilidad de los estudios para resolver los problemas cuantitativos básicos del planificador. El efecto empleo y otros efectos paralelos estimados aquí son al menos las variables cuyos niveles y relaciones es indispensable conocer como principios elementales en la toma de decisiones en este campo. La información abundante y el desglose minucioso de los cálculos que se presentan en los estudios a que se ha hecho mención dan buena pista para la realización de trabajos ulteriores de profundidad y refinamiento metodológico mayores. Asimismo, ante la queja frecuente de que la información es escasa, el conjunto de los estudios mencionados sugiere ideas interesantes sobre la prioridad de los datos para la investigación, y por lo tanto para su recolección. punto.

${ }^{26}$ Véase Boon, op. cit., respecto a todas estas cifras y las siguientes, sobre este

${ }_{27}$ Boon, op. cit., Cap. 3. Este cálculo supone los coeficientes de importación del cuadro insumo-producto de 1965. 
El problema habitacional en México, como en muchos países subdesarrollados, es en parte manifestación y en parte causa de la cadena de sucesos sociales encerrados en el concepto de bienestar. La distribución del ingreso es en mayor medida el eje de todos los aspectos relacionados con el nivel de vida, entre los cuales la vivienda es uno de los más destacados. La economía no proporciona poder adquisitivo de modo de hacer del consumidor un agente de estímulo automático a la construcción de vivienda, y, a su vez, la mala distribución del ingreso proviene de mecanismos sociales y económicos de concentración de la propiedad y los ahorros, falta de oportunidades educativas, imperfecciones del mercado, problemas estructurales básicos, etc. En otras palabras, el consumidor no adquiere vivienda porque carece de medios para hacerlo, y el sector de oferta no construye vivienda porque los márgenes de redituabilidad no le son atractivos, salvo en el caso de ciertos estratos de ingreso de número limitado. De aquí la inevitable consecuencia de que en los países subdesarrollados sea el sector público el encargado de corregir esta deformación, entre otras, y de reasignar los recursos para producir vivienda al alcance de grupos de ingreso bajo. Los intentos del sector público mexicano a este respecto han sido el resultado de presiones sociales de la naturaleza que se acaba de mencionar, pero la construcción de vivienda de interés social no ha formado parte de un plan más general. Esto ha sido en parte la razón de que los programas iniciados hayan tenido objetivos a corto plazo, de índole más bien paliativa, y que hayan funcionado dentro del marco financiero y técnico existente sin haber intentado estudiar algunos aspectos estructurales que obstaculizan la solución del problema.

El mercado de vivienda privado, es decr, aquel donde el gobierno no ejerce esfuerzos específicos, y que tiene como intermediario principal el sistema hipotecario y financiero, no puede más que tener alcances modestos, según puede inferirse del número de personas con solvencia suficiente para ser sujeto de las operaciones. En una muestra de hipotecas llevada a cabo para los estudios que se comentan puede verse la selectividad del financiamiento privado en cuanto a la solvencia del comprador de vivienda. En el financiamiento privado las operaciones de vivienda suelen realizarse con familias cuyo ingreso mensual es superior a 2300 pesos. $^{28}$ Según algunos observadores, esto se debe en gran parte a la falta de experiencia de las instituciones en operar con estratos de ingreso bajo. Desde luego que tales operaciones se concentran además en el medio urbano, ya que son prácticamente inexistentes en las áreas rurales. En este medio, al panorama de escasez y deterioro de la vivienda - aun descontando el hecho de que la migración campo-ciudad traslado parte del problema a otro medio donde su solución es más costosa y complicadase añade el descuido del sector público en cuanto al suministro de servicios sanitarios y otros. ${ }^{29}$

En resumen, el esquema de prioridades productivas refleja en el renglón de habitación el funcionamiento de una economía que produce bienes que satisfacen necesidades menos básicas que la vivienda, y el pano-

28 Ver Strassman, Empleo y alternativas financieras..., op. cit., cuadro 1.

29 Es evidente que se construye un monto apreciable de vivienda mínima y de bajo costo sin intervención de mecanismos financieros, sobre todo en las zonas urbanas marginales y en las áreas rurales. Por lo general, no existen planes ni programas organizados para fomentar o coordinar estos tipos de construcción. 
rama es criticable más bien porque su magnitud ha llegado a niveles donde la solución es de gran complicación, costo y duración.

Para atacar el problema, el gobierno mexicano ha establecido varios organismos y ampliado las funciones de otros ya existentes.

Los programas de construcción de vivienda en México se iniciaron a fines de los años cuarenta. En 1954 el gobierno creó el Instituto Nacional de la Vivienda, que operó en forma modesta hasta la introducción de varias reformas legislativas a principios de los años sesenta. En este período, instituciones públicas tales como el Instituto de Seguridad y Servicios Sociales de los Trabajadores del Estado (ISSSTE), el Instituto Mexicano del Seguro Social (IMSS), el Banco Nacional Hipotecario Urbano y de Obras Públicas (BNHUOP) e instituciones bancarias privadas emprendieron operaciones de mayor alcance en la construcción de vivienda y obras urbanas. En 1962 se creó el Programa Financiero de la Vivienda, en el Banco de México, que fue constituido por dos fideicomisos: el Fondo de Operación y Descuento Bancario a la Vivienda (FOVI) y el Fondo de Garantía y Apoyo a los Créditos para la Vivienda (FOGA), uno para completar y apoyar las operaciones bancarias en vivienda a mayores plazos y menor interés, y otro para garantizar los préstamos hipotecarios del primero, en ambos casos con relación a viviendas de bajo costo.

Con base en la Constitución de 1917, la legislación obrera mexicana establecía la obligación de los patrones de proporcionar vivienda a sus asalariados de acuerdo con ciertas circunstancias de antigüedad, ubicación y tamaño de las fábricas. Una iniciativa presidencial de diciembre de 1971 resultó en una modificación a la legislación, publicada en el Diario Oficial cinco meses después, en abril 24 de 1972, y que disponía la creación de un Instituto del Fondo Nacional de la Vivienda para los Trabajadores (INFONAVIT), cuya función es la de financiar y coordinar la construcción de viviendas para obreros y la de reparar y ampliar las existentes, además de dar crédito para el pago de adeudos anteriores contraídos por concepto de vivienda. Este Instituto, que inició sus operaciones de inmediato, integra sus recursos con una contribución patronal del $5 \%$ sobre los salarios ordinarios de los trabajadores en servicio (deducible de impuestos), más una contribución del gobierno federal cifrada el primer año en $\$ 2000$ millones.

El Instituto otorgará créditos al trabajador para construir o ampliar su vivienda, o cubrir adeudos previos por estos conceptos. ${ }^{30}$ Este organismo pretende realizar un programa de construcción de vivienda en gran escala, de un valor de $\$ 5000$ millones al año para 100000 unidades de vivienda de bajo costo, y sus implicaciones nacionales son de gran trascendencia, pues se procura hacer asequible la vivienda, en algunos casos mediante subsidio parcial, a la tasa de interés del $4 \%$ y en pagos de 10 a 20 años, a asalariados de muy bajo ingreso. "Por ejemplo, un trabajador que gana salario mínimo podrá adquirir una casa de 30 mil pesos con un abono de 190 pesos al mes; el trabajador con un ingreso de 1800 pesos mensuales, una de $50 \mathrm{mil}$ con 325 pesos de abono mensual. Se combate en esta forma el dramático error de emprender programas de vivienda fuera del alcance económico de los grupos obreros mayoritarios".31

30 Ver los detalles en Instituto del Fondo Nacional de la Vivienda para los Trabajadores, Disposiciones legales, México, 1972.

31 INFONAVIT, Asamblea constitutiva, Palabras del Director General, licenciado Jesús Silva Herzog F., México, 1972, pp. 30-31. 
En programas de esta naturaleza surgen de inmediato los dos objetivos obvios de atacar el déficit de vivienda y de crear empleo. La importancia relativa de estos objetivos no es cuestión de decisión arbitraria, sino que, al tratar de implementar una política, debe tomarse en cuenta la relación entre ambos. Esta política obliga a hacer consideraciones sobre la forma óptima de cumplir los objetivos, teniendo en cuenta los efectos negativos que pueden acompañar a una reasignación de los recursos, como es el caso de cualquier política de subsidio. Entre otras cosas, al hacer una evaluación de costo/beneficio de los resultados, deben considerarse la política de financiamiento del programa y la magnitud en que los fondos disponibles se asignan a este fin y no a otros. La creación del Instituto mencionado se expresó precisamente en estas direcciones, buscando la solución de ambas metas. El tipo de vivienda que se pretende construir es semejante a algunos de los tipos de bajo costo que se describen en los estudios que se comentan. El tamaño de la vivienda anunciado es en promedio de $50 \mathrm{~m}^{2}$, o de un costo medio de $\$ 50000$.

De las estimaciones del cuadro 9 sobre los contenidos de empleo pueden derivarse algunas conclusiones sobre el monto del empleo que generaría la construcción de 100000 viviendas, de superficie media de $50 \mathrm{~m}^{2}$, suponiéndola del tipo unifamiliar de bajo costo. La razón para seleccionar este tipo es que en la práctica es poco probable que en las áreas urbanas se construyan unidades unifamiliares del tipo mínimo, y por otra parte los tipos mediano y de lujo no se apegan a los criterios de definición de viviendas de interés social. El empleo que se generaría en la situación anterior sería de aproximadamente 126000 hombresaño, repartidos en 74000 de empleo directo y 52000 de empleo indirecto. $^{32}$

Este empleo de 126000 personas al año representa alrededor del $1.5 \%$ de la fuerza de trabajo urbana total del país en 1972, esto es, aquella que vive en localidades de 2500 y más habitantes. Representa también un $25 \%$ del incremento probable de la fuerza de trabajo total del país estimado para 1972. La parte de empleo directo, de 74000 personas, constituye más del $10 \%$ de la fuerza de trabajo de la construcción global (construcción e instalaciones), y un $27 \%$ del incremento anual neto estimado de la fuerza de trabajo urbana del país. La importancia del empleo que se puede generar con este volumen de vivienda resulta obvia si se considera el desempleo en el sector construcción, el cual, como se indicó con anterioridad, es de alrededor de 70000 personas, teniendo en cuenta solamente el desempleo abierto reflejado en las cifras censales de 1970. Es decir, el programa permitiría, al menos en teoría, absorber casi el $100 \%$ del desempleo así estimado.

Por su parte, el efecto empleo indirecto, generado por el programa de vivienda del INFONAVIT, de 52000 personas, incide en mayor medida sobre los sectores agropecuario, de servicios, comercio, extractivo y de transportes, en este orden, y depende, según se explicó, de la cantidad de empleo contenida en los materiales. Sobre esto debe hacerse hincapié en que el uso de los materiales puede representar un potencial importante de creación de empleo indirecto.

Todos estos efectos se realizan teniendo como causa inmediata el programa de construcción de vivienda, y por lo tanto la continuidad de la

32 Véase Boon, op. cit. 
creación de empleo depende de la continuidad del programa. Si se mantiene el mismo nivel de 100000 unidades de construcción de vivienda durante el segundo año, no se creará nuevo empleo por encima de los 126000 hombres-año iniciales, sino que se empleará a los mismos trabajadores ya empleados el primer año. Por otra parte, existe una relación de proporcionalidad directa en los efectos, de manera que un programa del doble de la magnitud del anterior crearía aproximadamente el doble del empleo. Todo esto sucede además dentro de los supuestos de uso de ciertas tecnologías, cuya selección puede hacer variar estas estimaciones.

Estas estimaciones gruesas señalan la importancia de las decisiones sobre tipos y formas de financiamiento en relación con la creación de empleo. Como se ha visto, el mecanismo de sustitución entre capital y mano de obra constituye el punto clave en la intensidad del uso del trabajo, y la selección del tipo de producto abarca esta dimensión así como la del mercado.

Las políticas de salarios mínimos en el país parecen haber empujado al empresario de la construcción en la dirección de desplazar trabajo y adoptar técnicas más intensivas de capital. La elección de tecnología, como mecanismo de evasión al costo del trabajo, puede representar una proporción importante del empleo generado. Por otra parte, la composición del producto de la construcción parece haberse modificado precisamente en la dirección opuesta a la creación de empleo. Todo esto lleva a considerar que la política salarial, por una parte, puede tener efectos negativos si no se refuerza en el sentido de impedir una formación de capital inadecuada del sector. Si el trabajo se encarece con relación al capital, y se deja abierta la posibilidad de adoptar nuevas técnicas, entonces la política de salarios resulta contraproducente con la creación de empleo. Según los cálculos sobre el efecto de mecanizar la excavación, el mezclado de cemento y el transporte vertical interno de materiales, el empleo generado en la construcción de vivienda de bajo costo puede resultar en una variación de $4.4 \%$ en el caso del tipo unifamiliar de bajo costo hasta $9.1 \%$ en el tipo multifamiliar también de bajo costo, ${ }^{33}$ equivalente a 6000 a 11000 empleos por año.

Acerca de la creación de empleo, debe mencionarse también que la forma de contratación del obrero, sujeta a una rotación elevada, implica que no existe un compromiso laboral de parte de los patrones que garantice el empleo estable, y por lo tanto la política de formación de capital recibe la influencia de este factor. Si existiera un gremio obrero con poder de negociación, no resultaría tan fácil modificar la composición del equipo de capital, y los intereses del obrero en este sentido quedarían más protegidos. La existencia de uniones sindicales no parece haber influido sobre estos fenómenos. Más bien su papel ha sido "encarecedor" del trabajo y de presión política en otros contextos. La defensa de los intereses del obrero de la construcción no puede obviamente hacerse sólo en términos de reforzar una política de alza de los salarios, en especial si una consecuencia inmediata de ello puede ser la del desempleo. El coeficiente de sustitución permite apreciar la sensibilidad del empresario en esta dirección, y en los estudios que se comentan se encuentra que es considerable.

33 Véase Boon, op. cit. 
La composición del producto es otro factor básico en la creación de empleo, y básico también en la ampliación del mercado, o lo que es lo mismo, en la satisfacción del déficit. La tendencia hacia la construcción de vivienda cara, como se infiere de los estudios, conduce a crear menos empleo por unidad de gasto y a impedir la adquisición de vivienda por parte de los grupos de ingresos bajos. La clave de las políticas de modificación de esta circunstancia se encuentra, pues, en el mercado de vivienda, donde las variables básicas son el precio y las condiciones de pago. Respecto del precio, el fenómeno de especulación de la tierra, tan agudo en los medios urbanos, ha encarecido en forma excesiva el costo de los terrenos, sobre todo en las grandes ciudades, y esto, por supuesto, ha alejado más de la posibilidad de adquirir vivienda a los estratos de ingresos bajos, y ha elevado las necesidades de recursos para los programas.

Las políticas de subsidios a la compra de vivienda pueden ir, como se señala en uno de los estudios, desde su recuperación total, dentro de plazos establecidos, hasta el donativo completo de la vivienda. Puede haber recuperación parcial, si se dona el costo del terreno, y la efectividad del plan de subsidios depende de la velocidad de recuperación, que permite recanalizar los fondos recuperados y, desde luego, del monto del subsidio. En todo caso, debe buscarse un óptimo de velocidad de recuperación, creación de empleo y cobertura. ${ }^{34}$ Una política de subsidio financiero a la vivienda debe limitarse a las viviendas de bajo costo, pues si se destina a las de mayor costo, los beneficiarios quedan en posibilidad de destinar sus propios recursos a objetivos de menor prioridad social. No necesariamente los planes financieros implican subsidio; en algunos, basta alargar el plazo de amortización y disponer abonos anuales crecientes.

Debe insistirse en que, en relación con una política de vivienda de bajo costo, es indispensable considerar medidas destinadas a evitar la especulación en terrenos, puesto que el costo de los mismos suele ser el renglón de mayor incidencia en el precio de venta de la vivienda. Por ejemplo, del cuadro 8 se deduce que el terreno representa desde el $26 \%$ del precio de venta total en el caso de viviendas unifamiliares de tipo mediano hasta $33 \%$ en las de tipo mínimo. En el caso de viviendas multifamiliares, el terreno representa entre el 8 y el $10 \%$ del precio de venta. En consecuencia, una política de adquisición de terrenos a bajo precio por entidades públicas -como el INFONAVIT en México- puede contribuir en alto grado a hacer asequible la vivienda a los sectores de la población de baja capacidad adquisitiva y promover así el objetivo simultáneo de crear mayores volúmenes de empleo.

La velocidad de la construcción es también otro factor determinante en cuanto a la creación de empleo, pero esto cae dentro del contexto de las presiones que los programas de construcción pueden ejercer sobre la economía. Este contexto es el inflacionario. La captación de fondos para la inversión y su reasignación tienen efecto inflacionario mixto, por el lado de los costos y por el de la demanda. La falta de capacidad de los sectores que abastecen la construcción para hacer frente a un aumento súbito de la demanda puede hacer subir los precios y ocasionar también un uso de técnicas más intensivas de capital. El efecto inversión, como

34 Véase Strassman, Empleo y alternativas financieras..., op. cit. 
se ha visto, es de magnitud considerable, y no puede olvidarse el hecho de que los sectores accesorios a la construcción deben contar con mecanismos flexibles de financiamiento del crecimiento de estas empresas. Sobre este efecto inversión debe aclararse de nuevo, igual que en el caso de las importaciones, que en los estudios que se comentan no se tomó en cuenta el uso de la capacidad instalada, en vista de la falta de información sobre este punto. Por esta razón, las estimaciones del efecto inversión conservan el margen de utilización de la capacidad subyacente en las relaciones inversión-producto utilizadas en el cálculo. 